\title{
COMPARISON OF SONOURETHROGRAPHY WITH CONVENTIONAL RETROGRADE URETHROGRAPHY FOR DETECTION OF MALE ANTERIOR URETHRAL ABNORMALITIES: A PROSPECTIVE STUDY
}

\author{
Jagadish G. Sutagatti ${ }^{1}$ Balakrishna B. V²
}

${ }^{1}$ Associate Professor, Department of Radiodiagnosis, Karnataka Institute of Medical Sciences, Hubli.

${ }^{2}$ Ex-Professor \& HOD, Department of Radiodiagnosis, Bangalore Medical College and Research Institute, Bengaluru.

\section{ABSTRACT}

\section{BACKGROUND}

The male urethra is a common site of strictures and congenital anomalies. Urethritis and pathologies of paraurethral structur es are also commonly encountered. Previously, conventional Retrograde Urethrography (RGU), antegrade urethrography and voiding cystourethrography were the standard imaging studies for the urethra. However, these techniques have various limitations and also carry the risk of radiation. Of late, the technique of ultrasonography known as Sonourethrography (SUG), a dynamic 3-dimensional study that can be repeated easily without ionizing radiation to the gonads has gained momentum.

\section{AIMS AND OBJECTIVES}

The objectives of our study were to find out the role of SUG in the evaluation of male anterior urethral lesions and to compa re the sensitivities of ascending urethrography and SUG in the detection of anterior urethral lesions.

\section{METHODOLOGY}

We conducted a prospective study over a period of 1 year; 40 male patients with voiding difficulties pertaining to the anterior urethra were selected. They were initially examined by conventional RGU followed by SUG. A catheter with a syringe loaded with normal saline was placed in the distal urethra and saline was gradually injected while examining the penis during SUG by a linear transducer of frequency 7.5-10MHz. The sonographic unit used was Esaote Biomedica AU5. The findings were subjected to tests of significance. The percentage sensitivities of both methods were calculated and compared.

\section{RESULTS}

Out of the 40 cases studied, 25 showed abnormalities on SUG and only 23 showed abnormalities on RGU. The stricture detection rates were $100 \%$ by SUG and $83 \%$ by RGU. The urethritis detection rates were $100 \%$ by SUG and $90 \%$ by RGU. The diverticulae detection rates were equal in both. The periurethral cyst detection rates were $100 \%$ by SUG and $50 \%$ by RGU.

\section{CONCLUSION}

SUG is a better imaging modality than older modalities like RGU for the evaluation of anterior urethral abnormalities.

\section{KEYWORDS}

Abnormalities, Imaging, Male, Ultrasonography, Urethra, Urethral Stricture.

HOW TO CITE THIS ARTICLE: Sutagatti JG, Balakrishna BV. Comparison of sonourethrography with conventional retrograde urethrography for detection of male anterior urethral abnormalities: a prospective study. J. Evolution Med. Dent. Sci. 2016;5(8): 351-353, DOI: $10.14260 /$ jemds/2016/78

\section{INTRODUCTION}

Various diseases affect the male urethra. Strictures due to urethritis induced by sexually and non-sexually transmitted infection, trauma, congenital and iatrogenic causes are common. Pathologies of the paraurethral glands are also encountered. Previously, conventional Retrograde Urethrography (RGU) and anterograde urethrography along with voiding cystourethrography were the standard evaluation techniques for the male urethra.[1] However, they have several drawbacks and radiation hazards. [2] The crosssectional imaging technique of ultrasound is a routine method of evaluating the urinary tract.

Financial or Other, Competing Interest: None.

Submission 16-12-2015, Peer Review 11-01-2016,

Acceptance 18-01-2016, Published 27-01-2016.

Corresponding Author:

Dr. Jagadish G. Sutagatti,

Associate Professor

Department of Radiodiagnosis,

Karnataka Institute of Medical Sciences,

Hubli, Karnataka.

E-mail: drjagadishsutagatti@yahoo.com

DOI: 10.14260/jemds/2016/78
It is of late being increasingly used for evaluating urethral and periurethral tumours. It had been previously underutilised for the evaluation of urethral abnormalities. Ultrasonography of the anterior urethra known as Sonourethrography (SUG) offers a dynamic, threedimensional study that can be repeated easily without ionizing radiation to the gonads. [2] With this background, we conducted a study to evaluate the role of sonourethrography in the investigations of male anterior urethral abnormalities and to compare the sensitivities of ascending urethrography and sonourethrography in the detection of anterior urethral lesions.

\section{METHODS}

This was a prospective study conducted in adults aged 20 to 50 years over a period of 1 year in a tertiary referral hospital. The Hospital Ethical Committee approval was obtained. 250 cases with voiding difficulties were referred to our department during the study period. Out of these, 40 male patients with voiding difficulties pertaining to the anterior urethra were selected for the study.

Female patients and all patients with voiding difficulties pertaining to the posterior urethra were excluded from the 
study. Written consent was obtained from the patients and privacy was maintained throughout the examinations.

The selected patients were initially examined by conventional RGU followed by SUG. They were made to lie down on the ultrasonography examination table in supine position with extended hip and knee. A thin polythene catheter attached to a $20 \mathrm{ml}$ syringe loaded with normal saline was placed in the distal most part of urethra. The catheter was secured in place by applying pressure over the glans of the penis, by holding it between the left index and middle fingers of the examiner.

Saline was gradually injected while examining penis sonographically by linear transducer of frequency $7-15 \mathrm{MHz}$. The sonography unit used for our study was Esaote Biomedica AU5 with linear transducer of $7.5-10 \mathrm{MHz}$. The images were obtained in both sagittal and transverse sections by placing the transducer over both dorsal and ventral aspects of penis. The transperineal approach was used as and when necessary.

The findings of retrograde urethrography were recorded on routine conventional radiograms. The findings of sonourethrogram were recorded in the memory disc of the sonography unit. The findings of both examinations were tabulated in the format prepared for the study. The findings of the study were then subjected to statistical tests of significance. Unpaired ' $\mathrm{t}$ ' test was used. The sensitivities were statistically calculated. The findings of both examinations were analysed in terms of percentage sensitivities of pathology detection rates.

\section{RESULTS}

Out of the 40 cases studied, 25 cases showed abnormalities on SUG, whereas 23 cases showed abnormality on RGU. The abnormality detection rate by SUG (63\%) was better than the detection rate by RGU (58\%). Out of the 25 abnormalities detected by SUG, 18 were strictures (Figure 1), 10 were urethritis cases (Figure 2), 1 was a diverticulum (Figure 3) and 2 were periurethral cysts (Figure 4).

Out of the 23 abnormalities detected by RGU, 15 were strictures (Figure 5), 9 were urethritis, 1 was a diverticulum (Figure 6) and another 1 was a periurethral cyst, thus showing that RGU failed to detect 3 cases of stricture, 1 case of urethritis and 1 periurethral cyst. Thus, the stricture detection rates were $100 \%$ by SUG and $83 \%$ by RGU. The urethritis detection rates were $100 \%$ by SUG and $90 \%$ by RGU. The diverticulum detection rates were $100 \%$ by SUG and $50 \%$ by RGU. (Table 1 ).

\section{DISCUSSION}

The length of strictures detected by SUG in our study was usually more than the length detected by conventional RUG. Similarly, Jack W et al.[3] and Gupta et al.[4] in their studies compared the length of strictures assessed by both the imaging modalities (SUG, RGU) and open urethroplasty. They found that SUG was consistently more accurate as compared with conventional urethrography and the stricture length was more in SUG measurements.

Clifford et al.[1] in a study found that SUG findings were as diagnostic as roentgen findings in 19 patients with urethral stricture and found that in 1 patient, SUG identified a bulbar urethral stricture which was not seen in RGU. In our study on 40 patients, SUG identified 3 strictures not demonstrated on RGU. In fact in two of our study cases,

RGU could not be performed, as the stricture was total, quite long and beginning from the meatus itself. Hence, we performed SUG by pushing saline by keeping the catheter tip at the meatus, while holding the glans closed for distal delineation of the extent of stricture. We instructed the patients to strain for demonstration of the proximal extent of the stricture by delineating the proximal urethra by urine itself.

Our study had some limitations. We did not do blinding of RGU results and did not correlate our results with intraoperative findings. Chaudhari et al..[5] did a similar study wherein the sonologist was blinded to the findings of RGU and the parameters studied were compared with the intraoperative findings. They found that in the estimation of strictures $4 \mathrm{~cm}$ long, RGU showed a lower sensitivity (60-80\%) compared with SUG (73.3-100\%).

In our study, we did not delineate strictures according to their lengths. Some author.[6] have compared the strictures after grouping them into short segment, intermediate segment and long segment and found that the sensitivity for detection of short, intermediate and long segment strictures with SUG was $100 \%, 90.9 \%$ and $88.2 \%$ respectively. Some authors. ${ }^{[7]}$ have evaluated SUG for detecting the degree of urethral spongiofibrosis. The sensitivity of SUG in detecting anterior urethral strictures was $98.5 \%$ in their study, whereas it was $100 \%$ in our study.

While conducting our study we noted that SUG was able to detect the severity and length of the strictures, though we did not document them; nevertheless, some authors Narendra et al.[8] were able to grade the urethral strictures by SUG based on Chiou et al. classification.[9]

SUG thus enables quantitative criteria for appropriate selection of patients for surgery namely-identifying strictures too long for resection. Conventional procedures like retrograde/antegrade urethrography can only poorly define the length of an anterior urethral stricture and cannot define the depth of scar formation. This information is better obtained by SUG as seen by our study results.

We, therefore, conclude that SUG is a better tool than RGU for the detection of male anterior urethral abnormalities and we recommend it as a reliable technique for routine use.

\begin{tabular}{|c|c|c|}
\hline Character & SUG & RGU \\
\hline Pathology detection & 63 & 58 \\
\hline Strictures & 100 & 83 \\
\hline Urethritis & 100 & 90 \\
\hline Diverticulae & 100 & 100 \\
\hline Periurethral cysts & 100 & 50 \\
\hline
\end{tabular}

Table 1: Percentage sensitivities of SUG and RGU in detecting different pathologies

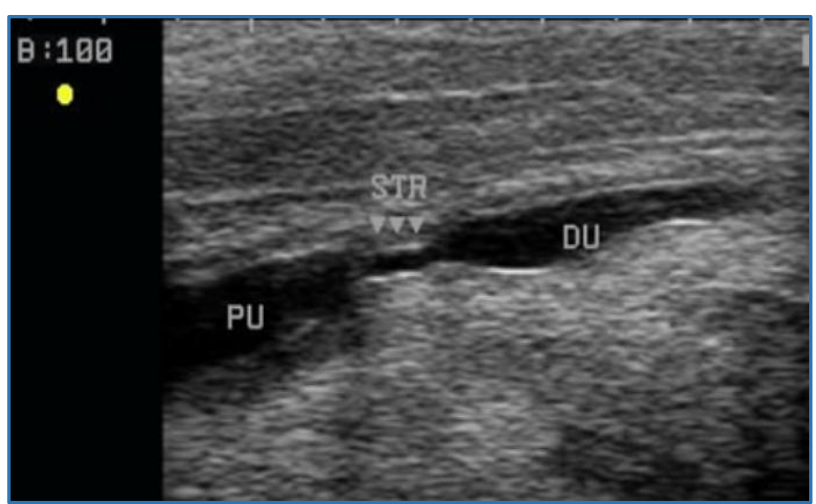

Fig. 1: Sonourethrogram showing narrow stricture at penile urethra associated with dilated proximal (PU) and distal urethra (DU) 


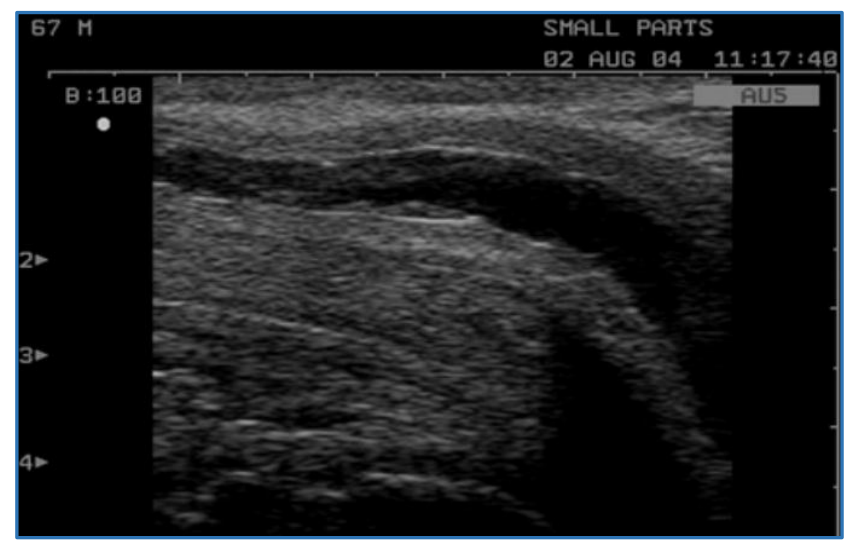

Fig. 2: Sonourethrogram showing urethritis (Littre's glands and irregular epithelium)

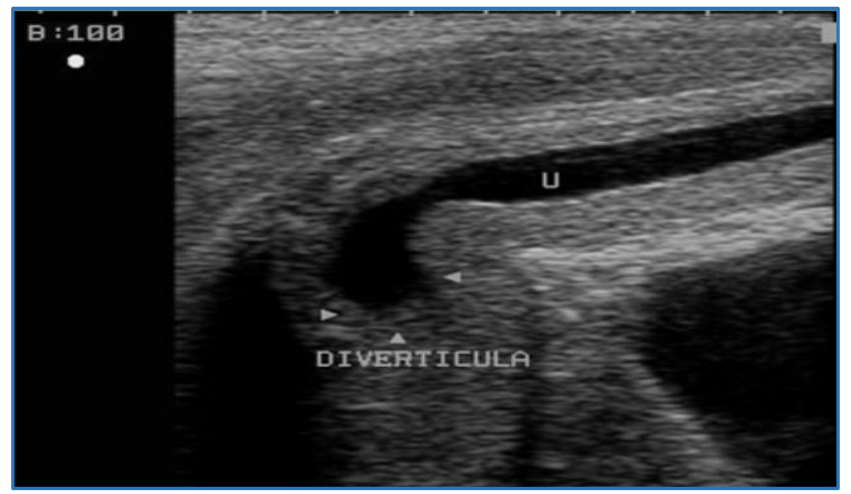

Fig. 3: Bulbo-membranous diverticulum on sonourethrogram

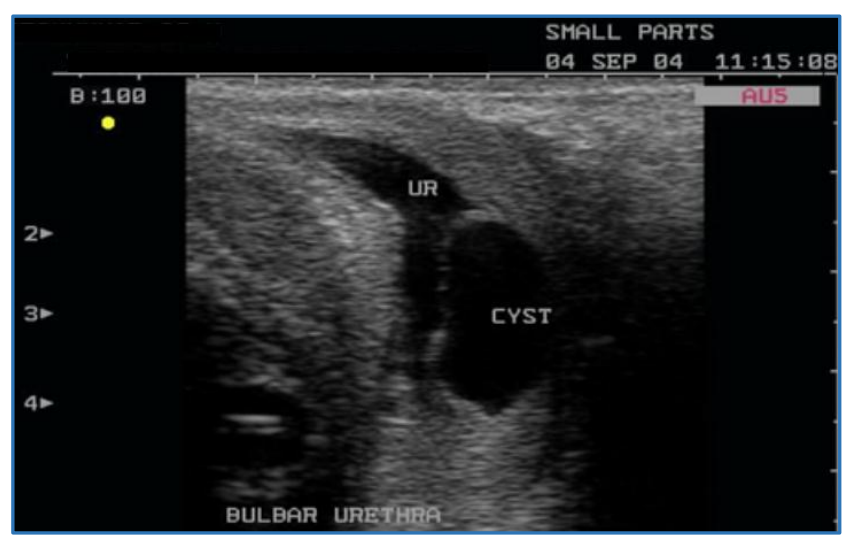

Fig. 4: Cowper's gland retention cyst on sonourethrogram appears as well defined cystic lesion separate from urethra at bulbo-membranous region

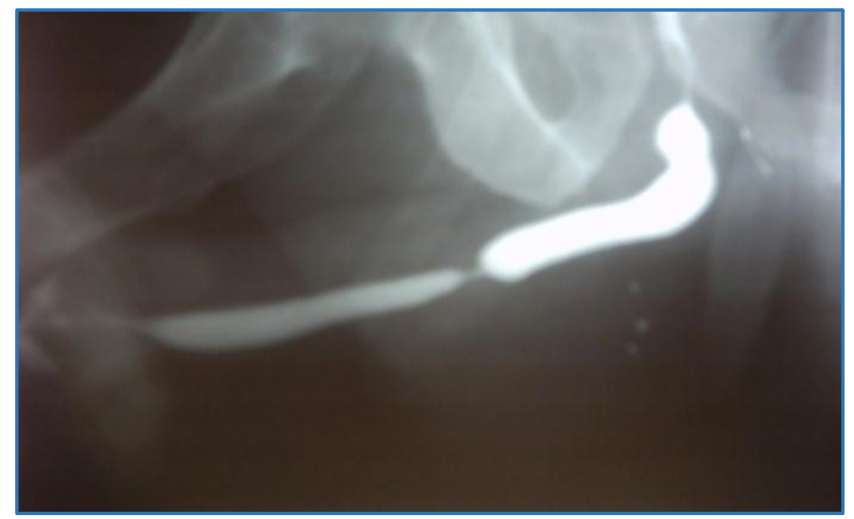

Fig. 5: Retrograde urethrogram showing narrow stricture

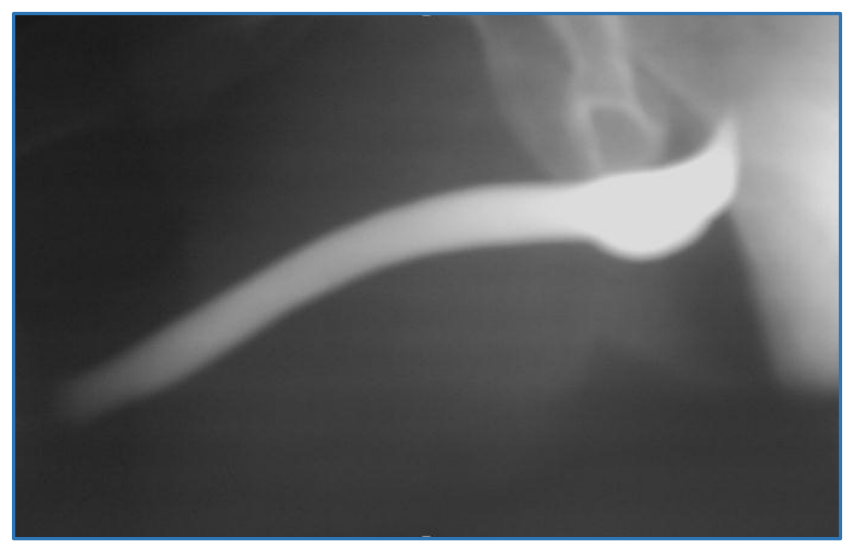

Fig. 6: Bulbo-membranous diverticulum on retrograde urethrogram

\section{REFERENCES}

1. Gluck CD, Bundy IA, Fine C, et al. Sonographic urethrogram: Comparison to roentogenographic techniques in 22 patients. Journal of Urology 1988;140:1404-08.

2. Klosterman PW, Laing FC, McAninch JW. Sonourethrography in evaluation of urethral stricture disease. Urologic Clinics of North America 1989;16(4):791-7.

3. McAninch JW, Laing FC, Jeffrey B. Sonourethrography in the evaluation of urethral strictures: a preliminary report. Journal of urology 1988;139:294-7.

4. Gupta S, Majumdar B, Tiwari A, et al. Sonourethrography in evaluation of anterior urethral stricture: correlation with radiographic urethrography. Journal of Clinical Ultrasound 1993;21:231-9.

5. Choudhary S, Singh P, Sundar E, et al. A comparison of sonourethrography and retrograde urethrography in evaluation of anterior urethral strictures. Clin Radiol 2004;59:736-42.

6. Mandal SK, Bhattacharyya SK, Mandal A, et al. Sonourethrography in the evaluation of anterior urethral stricture: correlation with retrograde urethrography in male. Int J Pharm Biomed Res 2012;3(2):77-80.

7. Sikarwar JS, Bhujade H, Muchhoria S, et al. Evaluation of anterior urethral pathology on sonourethrography and evaluation of degree of spongiofibrosis. JEMDS 2014;3(5):1206-14.

8. Tembhekar NG, Sonawane BD, Mashirkar A, et al. Sonourethrographic assessment of urethral obstruction. Journal of Evolution of Medical and Dental Sciences 2014;3(64):13971-78.

9. Chiou RK, Anderson JC, Tran T, et al. Evaluation of urethral strictures and associated abnormalities using highresolution and colour Doppler ultrasound. Urology 1996;47:102-7. 